\title{
Formation of subject composition and structure of innovative activity in the conditions of digitalization
}

\author{
Evgeniya Sizova ${ }^{1, *}$, Evgeniya Zhutaeva ${ }^{1}$, Olga Volokitina $^{1}$, and Vladimir Volokitin ${ }^{1}$ \\ ${ }^{1}$ Voronezh State Technical University, Moscow Avenue, 14, Voronezh, 394026, Russia
}

\begin{abstract}
The article is devoted to the application of the subject-oriented approach in the management of innovative activities in the context of digitalization. The key directions of the analysis of the readiness of the enterprise for the implementation of innovations are identified and substantiated and their impact on innovative processes is characterized. The multi-subject nature is noted as one of the most significant aspects of the implementation of innovative activities by an enterprise, which affects its effectiveness. A conceptual scheme for the interaction of subjects is proposed, taking into account the specifics of innovation and focus on improving its efficiency in key areas. In order to form the optimal subject composition, a classification of the participants in the innovation network is proposed. The mechanisms of accounting for the degree of workload of an innovative subject as a consequence of the multi-project nature of the activity are considered. An algorithm has been developed for constructing the optimal subjective structure of innovation activity, based on the use of elements of graph theory in order to assess its optimality. Risks of intersubjective interaction are formulated when participants of the external environment participate in the innovation network.
\end{abstract}

\section{Introduction}

Since digitalization is currently the main vector of economic transformation, market actors are faced with the task of choosing the most effective way to introduce digital technologies into business practices. One of the important aspects of digitalization for enterprises is the choice of business segments that provide the greatest benefits of the transition from traditional ways of organizing and implementing business processes to methods based on the use of digital advanced technologies. At the same time, the changes taking place at the enterprise should not adversely affect the state of the internal environment, since the reaction of the environment to certain processes of introducing technologies at early stages can cause the manifestation of destructive factors affecting the stability and integrity of the subject. To avoid the negative effect of digitalization, an enterprise must carefully analyze the specifics and possible consequences of changes in specific activities.

\footnotetext{
* Corresponding author: sizova@vgasu.vrn.ru
} 
Innovation activities carried out in the context of digitalization can be characterized as requiring a special approach when choosing management tools. The specificity of innovation activity is reflected in the works [1-3], where it is noted that the key features of innovation activity, which predetermine the choice of methods and management tools, are the complexity, high risk and uniqueness of the innovation implementation processes. As a result of the manifestation of these features, companies are forced to carry out activities to introduce innovations in a multi-project and multi-entity environment. Since enterprises that have switched to an innovative path of development do not function on their own, but in direct or indirect contact with other subjects of the innovation environment, the works [4- 6] note the expediency of applying a subject-oriented approach to innovation management. The issues of classification of subjects participating in innovative activity were considered in works [79], while in order to study the subject composition, the most common functional-role approach to differentiation of participants. The problems of the network approach to innovation was considered in the works [10-12], where the polycentric nature of the relationships is noted, which allows each participant, if necessary, to integrate into any network segment, which generates many options for subject interaction.

All of the above are factors that generate additional risks during the entire innovation process, which predetermines the need to develop an appropriate methodological toolkit for the formation of the subject composition and structure of innovation activity.

\section{Materials and Methods}

The development and implementation of innovations in the activities of an enterprise is an important management decision, which should be preceded by a thorough analysis of the level of innovative readiness. The following are key aspects that should be analyzed and assessed before making a final decision on recognizing an enterprise as ready for innovation: 1. The level of qualifications of personnel in the field of digital technologies and innovation. The complexity and multidimensionality of the procedures for managing innovation in the context of the spread of digital technologies requires persons involved in the development and implementation of innovations to have the appropriate qualifications, knowledge and skills. The presence in the structure of the enterprise of an effective innovation center with highly qualified managers and project executors will allow to more fully realize the innovative potential of the enterprise and reduce the number of erroneous decisions during the implementation of the project.

2. The level of adaptability of the internal elements of the enterprise. First of all, this concerns the organizational structure, since innovation is capable of causing structural changes both at the development stage and as a result of implementation. It may be necessary to create, merge, spin off, split business units, reorient information, financial, logistics flows and other similar changes, during which it is important to preserve the structural integrity of the enterprise. Another aspect is the state of the innovative corporate culture, which characterizes the general attitude and willingness to innovate. The more loyal the attitude to innovation within the enterprise, the more intensively the innovative potential of each employee engaged in the innovation process is manifested.

3. Satisfactory financial condition. The company must be prepared for the long-term withdrawal of significant amounts of financial resources from circulation, as this can cause problems with solvency within the planning horizon. In addition, when planning financial flows, it is necessary to take into account the likelihood of failure to achieve the planned financial results based on the commercialization of the innovation.

4. Possibility of innovative integration with other participants. In conditions of limited and uneven distribution of innovative resources, it may be necessary to establish relations with the subjects of the innovation infrastructure on the basis of formal or informal 
grounds. This is especially important in the absence of the subject's previous experience of independent implementation of innovations. The success of product or technology implementation and market demand will depend on the degree of coordination of the actions of all participating subjects, the completeness of the analysis of their innovative potential and the quality of its implementation in the course of creating an innovation.

5. The state of the information space. This may include the availability of a database on previously implemented projects, the availability of information on advanced developments in the required area, the level of development of the intellectual property protection system, the availability of specialized information technologies designed to interact during the implementation of the innovation process and processing of relevant information.

6. Sufficiency of material and technical resources. If the enterprise does not have the resources required to create an innovation, or in case of achieving greater benefits from using the resources of third-party organizations, the enterprise can resort to using outsourcing tools. The volumes of transfer are determined by the available and required resources, as well as the specific goals and objectives of innovation. However, when deciding on innovative outsourcing, in addition to direct financial benefits, it is necessary to take into account indirect strategic effects, such as the acquisition of knowledge and experience, the development and strengthening of the ecosystem, the growth of the organization's viability, and the development of an innovative corporate culture [13]. Full outsourcing can slow down their manifestation processes.

When implementing an innovation, it is necessary to take into account not only the specifics of the enterprise that generates the innovation. In the course of the innovation process, a significant number of subjects are involved, characterized by fundamental differences both in the nature of the impact on innovation and in the incentives for behavior and the mechanisms of intersubjective interaction. The main task in this case is the formation of such a composition of participants in order to fully meet the requirements of the innovative project, and at the same time provide all participants with a sufficient level of profitability from working as part of the innovation network. The effectiveness of interaction between the subjects involved in the innovation process, the degree of their interest in the results, the coordination of actions, the quality of communication channels between them will affect not only the success of achieving the planned results of the innovation project, but will also affect the efficiency of a significant number of enterprise subsystems.

For the purposes of this study, we differentiate the concepts of "subject composition" and "subject structure". Under the subject composition we mean the finite number of subjects involved in the implementation of the network innovation process and directly influencing the creation of innovation or promoting its implementation, development or diffusion. Under the subject structure we mean an ordered set of relationships between subjects, due to the need to implement the network innovation process.

\section{Results}

Let us define adaptability, invariance, ease of coordination and stability as the main requirements for the subject composition and structure of innovation activity. It is also necessary to remember that the interaction of subjects should ensure the lowest possible amount of transaction costs. Based on the analysis of the most common approaches to differentiating the subjects of innovation, taking into account the importance of recording digitalization processes that affect the production of innovations, and also focusing on the possibility of further application of the classification in order to optimize the subject structure of innovation, we proposed a conceptual scheme for the interaction of subjects of innovation (fig. 1). 


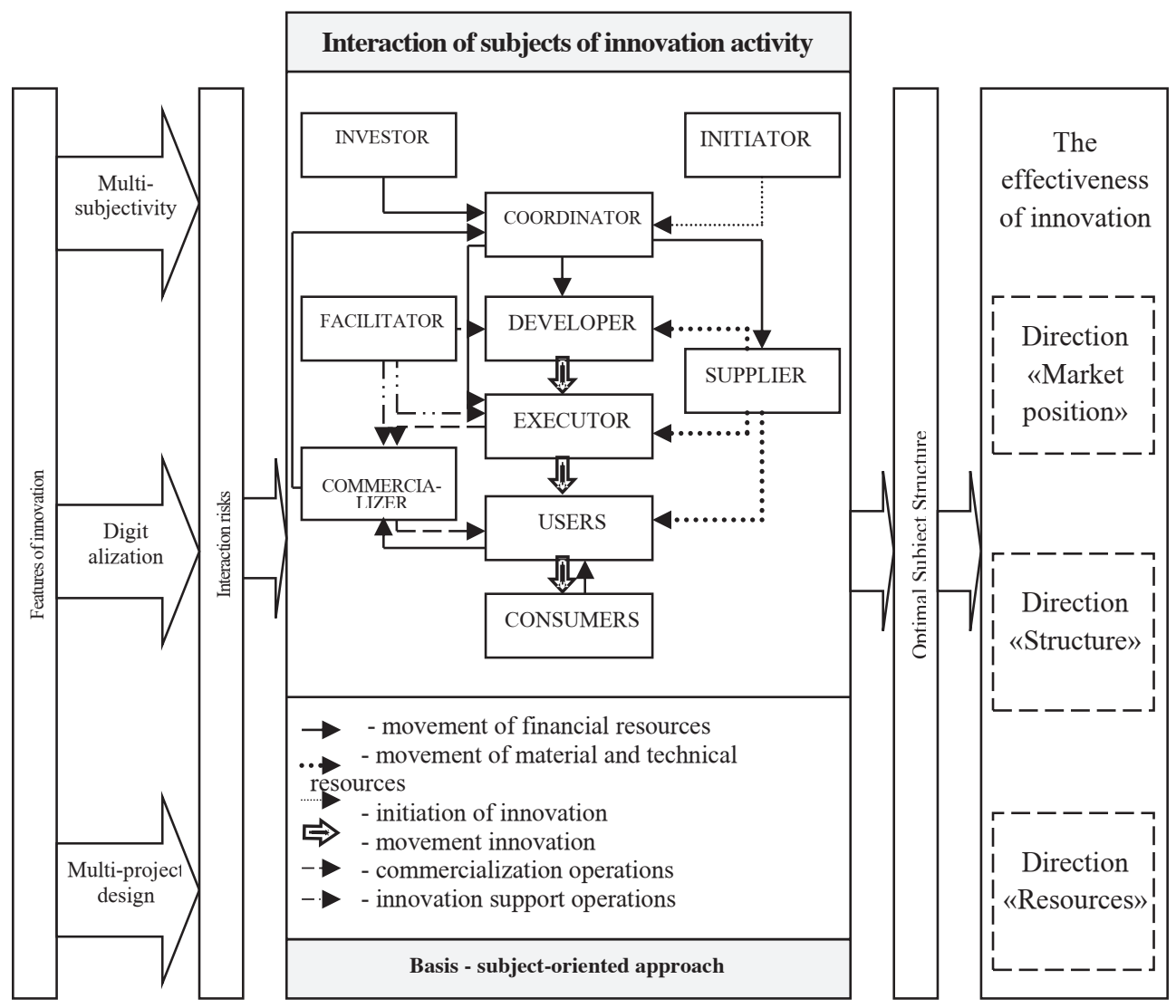

Fig. 1. Conceptual scheme of interaction between subjects of innovation activity

The innovation network formed as a result of the interaction of subjects as an ordered set of subjects organized according to the principle of heterarchy interacting with each other through special communication channels and exchanging resource and other flows in order to create innovative products, works, services, which contributes to the emergence of a network effect as a consequence of the economic effect of increasing returns [12].

The qualitative characteristics of the constituent entities of the network differ significantly both in terms of functional roles, statuses, types, and other attributes. Compared to hierarchical types of structures, it is not the position of a specific subject and not the relationship of leadership and subordination that is important, but the intensity of relationships with other actors, on which the timing and cost of project implementation will depend. The network approach to innovation is a compromise between self-organizing and controlled behavior, since at the same time it provides more freedom in the choice of methods and methods of conducting innovation, and at the same time imposes a number of restrictions due to the manifestation of the specifics of integration processes [14]. Therefore, the network approach requires the adaptation of existing procedures in terms of protecting intellectual property, planning target indicators for innovation and monitoring their achievement. So, for example, the basis for effective interaction of subjects should be sufficient legal security based on the use of tools that imply the formalization of relations in the field of permission, control and incentives.

The formation of the subject composition should be based on the account of all the features and factors that can multiply the risks of an innovative project. In order to form the 
subject composition, a classification of the participants in the innovation network is proposed (table 1) according to a number of features that are of particular importance in assessing the feasibility of including a subject in the composition of participants.

Table 1. Classification of participants in the innovation network.

\begin{tabular}{|c|c|c|}
\hline $\begin{array}{c}\text { Classification } \\
\text { attribute }\end{array}$ & $\begin{array}{l}\text { Types of participants in } \\
\text { the innovation network }\end{array}$ & Characteristic \\
\hline \multirow{2}{*}{$\begin{array}{l}\text { In relation to the } \\
\text { enterprise as a } \\
\text { single entity }\end{array}$} & $\begin{array}{l}\text { Subject of the external } \\
\text { environment }\end{array}$ & $\begin{array}{l}\text { Third party enterprise, engineering center, business } \\
\text { incubator, design bureau, etc. }\end{array}$ \\
\hline & $\begin{array}{l}\text { Subject of the internal } \\
\text { environment }\end{array}$ & $\begin{array}{l}\text { Subdivision, branch, subsidiary or dependent structure, } \\
\text { etc. }\end{array}$ \\
\hline \multirow{4}{*}{$\begin{array}{l}\text { Depending on the } \\
\text { degree of frequency } \\
\text { of entry into the } \\
\text { network }\end{array}$} & New subject & Never been involved in the network before. \\
\hline & $\begin{array}{l}\text { An entity that has already } \\
\text { participated in the } \\
\text { network }\end{array}$ & $\begin{array}{l}\text { One-time participation at any stage of the innovation } \\
\text { process. }\end{array}$ \\
\hline & $\begin{array}{l}\text { Subject periodically } \\
\text { involved in work }\end{array}$ & $\begin{array}{l}\text { Participation in a number of projects regardless of the } \\
\text { time of implementation. }\end{array}$ \\
\hline & $\begin{array}{l}\text { Permanent member of } \\
\text { the network }\end{array}$ & Participation in the ongoing project is provided by default \\
\hline \multirow[t]{2}{*}{$\begin{array}{l}\text { On a territorial } \\
\text { basis }\end{array}$} & Resident entity & $\begin{array}{l}\text { Meets the requirements for recognition as a resident } \\
\text { under applicable law. }\end{array}$ \\
\hline & Non-resident entity & $\begin{array}{l}\text { Does not meet the requirements for recognition as a } \\
\text { resident in accordance with the current legislation. }\end{array}$ \\
\hline \multirow{2}{*}{$\begin{array}{l}\text { Depending on the } \\
\text { number of subjects } \\
\text { at the stage }\end{array}$} & Monosubject & $\begin{array}{l}\text { The entrusted stage of the innovation process is } \\
\text { performed independently. }\end{array}$ \\
\hline & Group member & $\begin{array}{l}\text { The entrusted stage of the innovation process is } \\
\text { performed jointly with other subjects. }\end{array}$ \\
\hline \multirow{2}{*}{$\begin{array}{l}\text { Depending on the } \\
\text { impact } \\
\text { innovation }\end{array}$} & $\begin{array}{ll}\begin{array}{l}\text { Subject influencing } \\
\text { innovation }\end{array} & \\
\end{array}$ & $\begin{array}{l}\text { Generates innovation by influencing its quantitative and } \\
\text { qualitative characteristics. }\end{array}$ \\
\hline & $\begin{array}{l}\text { Subject accompanying } \\
\text { innovation }\end{array}$ & $\begin{array}{l}\text { Provides the work of generating entities or } \\
\text { organizational, informational, regulatory and other } \\
\text { support for innovation }\end{array}$ \\
\hline \multirow[t]{2}{*}{$\begin{array}{l}\text { Depending on the } \\
\text { staging }\end{array}$} & $\begin{array}{l}\text { Subject with one-step } \\
\text { participation }\end{array}$ & $\begin{array}{l}\text { Participates in the network at one separate stage of the } \\
\text { innovation process. }\end{array}$ \\
\hline & $\begin{array}{l}\text { Subject with multi-stage } \\
\text { participation. }\end{array}$ & $\begin{array}{l}\text { Participates in the network several times at different } \\
\text { stages of the innovation process. }\end{array}$ \\
\hline \multirow{3}{*}{$\begin{array}{l}\text { Depending on the } \\
\text { nature of the } \\
\text { movement of the } \\
\text { object } \\
\text { innovation }\end{array}$} & Recipient & Accepts the results from the previous stage. \\
\hline & Donor & Transfers the results obtained in the previous stage. \\
\hline & Channel & $\begin{array}{l}\text { Accepts the results obtained at the previous stage and } \\
\text { transfers its results to the next stage. }\end{array}$ \\
\hline
\end{tabular}

The diversity of innovation management structures is a consequence of differences in the composition of subjects, the degree of intensity of intersubjective interactions in the course of the innovation process, the presence or absence of outsourcers, the presence of duplication of functions and other characteristics. The choice of the optimal structure from the set, all other things being equal, will ensure not only the minimization of project implementation costs, but will also increase the effectiveness of activities in the strategic aspect.

Structuring innovation activity can be difficult due to the fact that a subject that involves participation in the innovation network can be simultaneously engaged in the implementation of a number of projects. If we are talking about a third-party entity, it independently determines the degree of readiness to participate in another project, since its further responsibility for the results is guaranteed by a concluded contract or other similar document providing for responsibility for non-performance of work. For internal entities that have a limited reserve of opportunities for participation, excessive overload can lead to a decrease 
in efficiency from participation, non-compliance with the declared deadlines for the completion of work or failure to achieve indicators.

The load on the subject at the considered moment of time $t$ can be estimated using the load factor, adapted by us taking into account the task (based on [15]):

$$
K_{t}=\frac{\bar{V}-\left(\sum_{a=1}^{n} V_{a}+V_{s}\right)}{\bar{V}}
$$

where $K_{t}$-subject utilization factor;

$\bar{V}$ - average workload of a subject for the entire period under consideration;

$\sum_{a=1}^{n} V_{a}$-total workload of a department in the implementation of $\mathrm{n}$ projects at a time $t$;

$V_{s}$ - the planned load from the participation of the subject in the implementation of project $s$ at the moment of timet.

In order to build an optimal subject structure, we have proposed a methodology for structuring innovation (fig. 2).

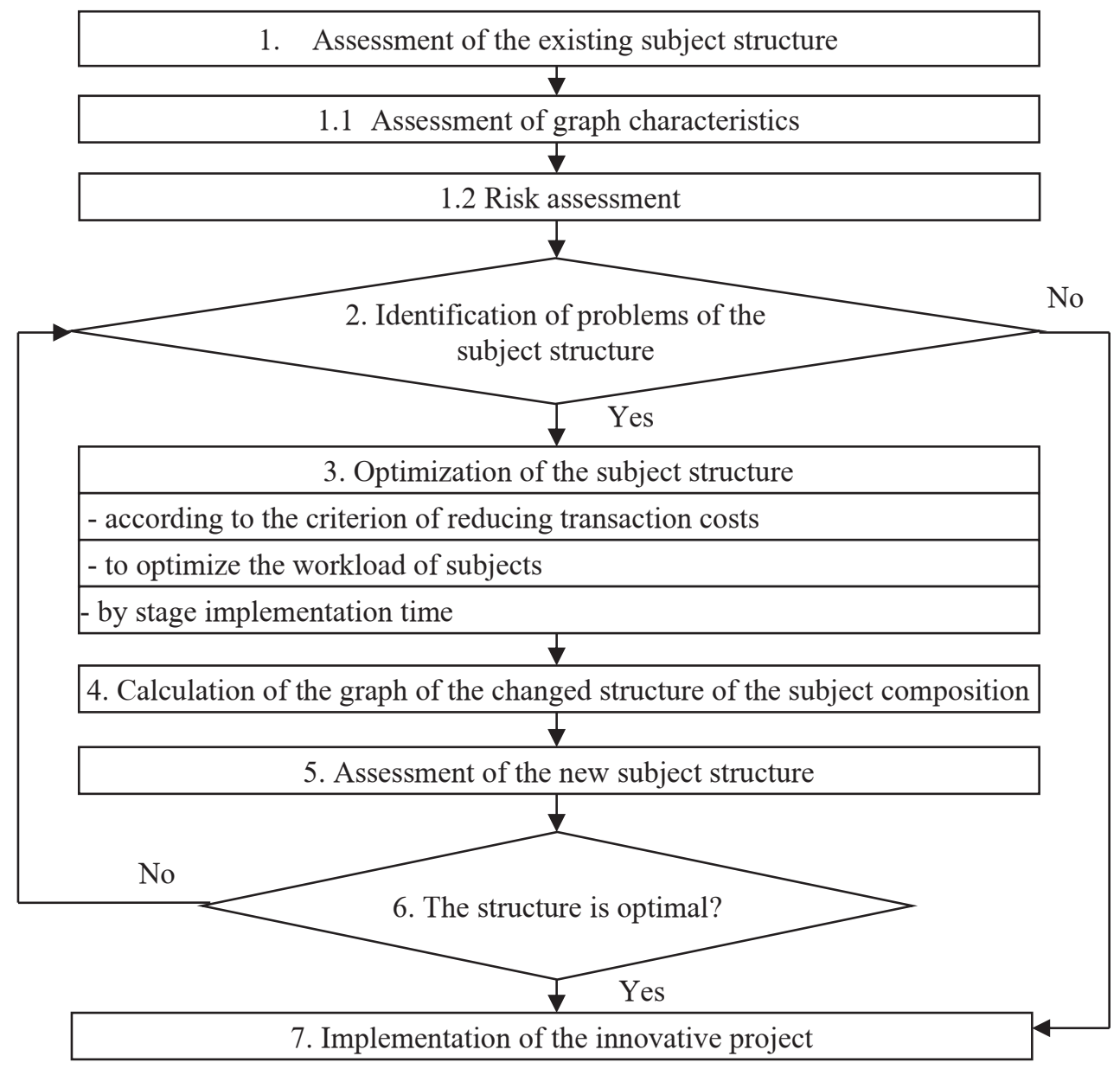

Fig. 2. Block diagram of the algorithm for constructing the optimal subject structure of innovation

The description of subject interactions in the structure of innovation management in the form of a graph allows providing an analytical opportunity to assess the nature of interactions between subjects from the point of view of the effectiveness of the functioning of the structure of the innovation network. Since the methods of graph theory are applicable to 
assess the subjective structure, at this stage, it is advisable to use the characteristics of unloaded graphs of the subjective structure (connectivity, density, centrality, diameter, structural redundancy, Euler cycles) as a basic assessment. At the stage of a more detailed assessment, we consider it expedient to use classical criteria for assessing graphs, modified on the account of the problem specifics under consideration. To assess the cost criterion in accordance with the provisions of the network planning and management methodology when calculating the graphs, the workload can be characterized by the stress coefficient. It represents the ratio of the duration of the trapped paths between the same events, one of which is the path of maximum duration passing through a given work, and the other is the critical path.

\section{Discussions}

Among the most common negative manifestations of interaction with external actors during the implementation of an innovation are the following:

- the distance of third-party enterprises from the internal environment, as a result of which the needs and peculiarities of activities may not be fully taken into account;

- increased susceptibility to the influence of macroenvironmental factors (political, social, regulatory, etc.)

- a significant increase in transaction costs, including all losses from ineffective decisions;

- the likelihood of complete or partial incompatibility of internal control systems as a result of insufficient efficiency of work on unification and standardization;

- informational isolation and barriers to obtaining relevant and reliable information about the goals, objectives and intentions of an external entity;

- temporary and functional "break" of the innovation process at the stage transferred to an external subject;

- conflict of information (communication) systems of subjects;

- transfer of key control functions to an external entity during the implementation of the stage of the innovation process, which can lead to inconsistency of management decisions.

All this leads to the emergence of specific risks of interaction. These risks include the following:

1. Default risks:

- risks of opportunistic behavior - imply a possible orientation of the subject to obtain their own benefits to the detriment of the effectiveness of the project;

- risks of changing the state of an external entity - provide for the entity's loss of economic independence or financial stability as a result of unforeseen factors, making it difficult or impossible to participate in the project;

2. Risks of conflicts of joint work - include both conflicts of management systems of organizations, and conflicts between team members or management;

3. Information (communication) risk - provide for the likelihood of incompatibility of the parameters of information flows, which can lead to the receipt of information out of time, not in full or with distortions;

4. The risk of interference in the internal environment - violation of the structural integrity of the enterprise;

5. Risks of a pure transaction - provide for the possibility of errors due to the complexity of the procedures for the intersubjective transfer of innovation at a particular stage of its creation, including errors in the conclusion of transactions, imperfection of valuation mechanisms, etc. [16]. 
When making a decision to involve subjects of the external environment in the implementation of an innovative project, it is necessary to carefully assess the level of interaction risks and compare it with the benefits of the subject's participation.

\section{Conclusion}

Maintaining the required level of enterprise competitiveness, ensured through the effective implementation of innovations, should be based on the creation of a favorable innovation climate, the use of progressive digital technologies, and the construction of a system for the movement of information flows in such a way as to ensure the efficiency of joint work of all involved persons and departments. With the intensification of innovative activities of companies in conditions of uncertainty and risk, the burden on management structures increases proportionately. Management of innovation as a continuous and complex process implies the use of appropriate methods and methods of management, taking into account the significant scale and pronounced strategic focus of innovation. The changes observed as a result of the implementation of innovations, provided that they are effectively managed, can go beyond the innovation-generating enterprises. They are able to affect all market participants, in turn, stimulating them to intensify innovation and to establish one-time or long-term partnerships, becoming elements of innovation networks of varying degrees of strength.

The effectiveness of the organization of innovative activities directly affects the effectiveness of innovation. Structuring innovation activity as one of the most important management aspects provides for the construction of a system of relationships between the participating subjects in such a way as to ensure the manifestation of a synergistic effect with minimal transaction costs. Each subject should assess the level of profitability from participation in the innovation network independently, determining the feasibility of such interaction. By implementing the entire innovative project entirely on its own, the company achieves certain strategic advantages in the form of experience and building up a knowledge base, but in some cases the effect of transferring individual stages of the innovation process to outsourcing can be much higher. In any case, the determination of the composition of the participants will influence both the achievement of the planned indicators of the implementation of the innovation and the efficiency of the enterprise as a whole.

\section{References}

1. Kh. Gumba, S. Uvarova, S. Belyaeva, S. Revunova, MATEC Web of Conferences, 106, 08023 (2017)

2. D. Basalaev, News Of TSU. Economic and legal Sciences, 3-1, 19-23 (2018)

3. I. Lukmanova, E. Sizova, E. Zhutaeva, O. Mironova, MATEC Web of Conferences, 239, 04014 (2018)

4. A. Gromov, Yu. Bilinkis, A. Fleshman, T. Novikova, E. Khudobin, D. Torshin, Business Informatics, 1(31), 18-30 (2015)

5. Kh. Gumba, S. Uvarova S., E. Prokhin, Ya. Andryunina, E. Sizova, S. Belyaeva. Management Of Innovative Eco-Building Projects Based On A Subject-Oriented Approach, Moscow (2019)

6. N. Przhedetskaya, E. Kulkova, V. Sheveleva, Fundamental Research, 8-1, 212-216 (2017)

7. L. Obolenskaya, Management Sciences, 4(13), 55-61 (2014) 
8. L. Erigina, K. Orlova, Siberian Journal Of Science And Technology, 4, 1113-1118 (2016)

9. N. Chernorizova, Innovation and investment, 4, 26-29 (2018)

10. J. Torkunova V., Fundamental research, 6, 1286-1289 (2014)

11. T. Badaraev, Bulletin of Tomsk State University. Economy, 2(34), 203-211 (2016)

12. I. Zaraichenko, Bulletin of the Kazan technological University, 3-16, 248-252 (2013)

13. E. Sizova, Paradigm Of Modern Science Through The Eyes Of Young People, 14, 409412 (2020)

14. I. Omelchenko, D. Lyakhovich, E. Gorlacheva, Science And Education: Scientific Publication Of Bauman Moscow State Technical University, 12, 1-17 (2010)

15. N. Titova, University Administration, 5-6(33), 143-151(2014)

16. E. Sizova, Economics and entrepreneurship, 3-1(80), 1005-1008 (2017) 